\title{
L'HOMME L'Homme
}

229 | 2019

Varia

\section{Georges Guille-Escuret, Structures sociales et systèmes naturels. L'assemblage scientifique est-il réalisable?}

Léo Mariani

\section{OpenEdition}

Édition électronique

URL : https://journals.openedition.org//homme/33711

DOI : 10.4000//homme.33711

ISSN : 1953-8103

Éditeur

Éditions de l'EHESS

\section{Édition imprimée}

Date de publication : 1 mars 2019

Pagination : 192-194

ISSN : 0439-4216

\section{Référence électronique}

Léo Mariani, « Georges Guille-Escuret, Structures sociales et systèmes naturels. L'assemblage scientifique est-il réalisable? », L'Homme [En ligne], 229 | 2019, mis en ligne le 01 mars 2019, consulté le 07 janvier 2022. URL : http://journals.openedition.org//homme/33711 ; DOI : https://doi.org/10.4000//homme 33711

(ㄷ École des hautes études en sciences sociales 
Georges Guille-Escuret

Structures sociales et systèmes naturels.

L'assemblage scientifique est-il réalisable?

Londres, ISTE Éd., 20I8, 233 p., bibl., index («Sciences, société

et nouvelles technologies. Série interdisciplinarité autour du social» 3 ).

$C^{\prime}$

EST ASSURÉMENT à un exercice ambitieux et offensif que se livre ici Georges Guille-Escuret. Ambitieux parce qu'il vise large: il s'agit de refonder l'épistémologie de l'interdisciplinarité socio-écologique. Mais aussi parce que les exigences d'efficacité que l'auteur s'impose dès la quatrième de couverture laissent peu de place aux dérobades : "ce livre sera une réussite si, et seulement si, il constitue un début». L'offensive tient au fait que Georges GuilleEscuret opère une revue sans concession "des causalités caricaturales du déterminisme biologique» d'une part, et de celles du relativisme que les sciences humaines et sociales lui opposent en général, d'autre part. Si les anthropologues s'adonnent à la première critique presque rituellement, force est de constater que la seconde est rare de nos jours. Elle n'en est que plus dérangeante. Georges Guille-Escuret montre ainsi comment le "vrai idéalisme» qui domine selon lui l'anthropologie s'est construit en opposition à un «faux matérialisme» (darwinisme social en tête), dont il craignait à juste titre les dérives, mais dont il a trop vite inversé les excès, substituant la cause (et le causalisme) discutable du signe à celle, tout aussi contestable, de la biologie. À terme, on se convainc que l'anthropologie a ainsi introduit un curieux 
amendement moral dans ses fondements épistémiques (pp. 24 et 208) et que, sur ce point, le "matérialisme méthodologique» humble (revendiqué) et concret défendu par Georges Guille-Escuret contient les germes d'une émancipation. Peut-on faire de l'anthropologie et développer une pratique interdisciplinaire en tenant compte des matériaux, des cochons sauvages, des cépages, des molécules (etc.), tout en se permettant de déborder les subjectivités humaines, les idées et les esprits? La question est d'une vibrante actualité, mais la réponse apportée par Georges Guille-Escuret se démarque franchement des conventions. C'est logiquement face à sa propre discipline que l'auteur se montre le plus combatif et, même si l'envergure interdisciplinaire de son propos ne fait aucun doute, tout porte à penser que c'est aux anthropologues qu'il s'adresse en premier lieu. La critique est frontale, parfois généralisatrice et volontiers provocante, mais il faut reconnaître que les motifs de l'interpellation sont aussi modestes que légitimes: est-il possible, pour l'anthropologie, de s'affranchir de la tutelle équivoque de la philosophie, en abandonnant ses prétentions métaphysiques pour s'attacher à l'élaboration et la résolution de problèmes appliqués, avec des disciplines dont les savoirs sont pratiques et non réflexifs?

Il est certain que Georges Guille-Escuret a les moyens de ses ambitions, de par sa formation (il est titulaire d'une thèse en anthropologie et d'une autre en zoologie), mais aussi parce que Structures sociales et systèmes naturels est le point d'orgue d'une imposante bibliographie (qui débute avec une étude exhaustive sur l'histoire viticole des Corbières, puis passe, entre autres, par une exploration des Sociétés et leurs natures, trois volumes sur le cannibalisme et un essai sur l'écologie $\left.{ }^{1}\right)$. De cet itinéraire, il ressort une direction cardinale, un sillon revêche qui ramène obstinément à la surface les refoulements de l'anthropologie, en particulier les matériaux, l'histoire et l'événement. Il faut lire ce nouvel opus à la lumière de ces fondements, et de sa filiation avec une nébuleuse d'auteurs englobant Haudricourt,
Leroi-Gourhan, Barrau et Creswell, dont Georges Guille-Escuret est aujourd'hui l'un des derniers porte-parole authentiques. Au fond, soutient-il, cet héritage serait subsidiaire si les problèmes qu'il pose ne se posaient plus, ou s'ils avaient été scientifiquement invalidés. Georges Guille-Escuret s'attache au contraire à montrer leur actualité, arguant que ces problèmes souffrent d'une indifférence que seule leur position dominée dans le champ de l'anthropologie peut vraiment justifier (position matérialisée par le dédain poli que ces théories atypiques, "rustiques» selon ses termes, ont suscité chez les tenants de l'orthodoxie disciplinaire). Au vu du contraste entre l'intérêt des questions et leur influence, on peut difficilement ne pas le suivre sur ce point. Les bonnes problématiques ne survivent pas toutes, et les sciences sociales n'échappent pas aux logiques d'assemblage et d'exclusion qu'elles étudient chez les autres.

L'ouvrage est organisé en quatre chapitres denses et directs. Dans le premier, Georges Guille-Escuret pose les jalons d'une stéréoscopie (technique permettant de produire un relief à partir de deux images planes) scientifique, qui constitue la base soigneusement argumentée de ses ambitions épistémiques. Il s'agit, pour le dire forcément trop vite, de développer des solutions scientifiques capables de passer l'épreuve du terrain, une qualité que, selon l'auteur, la plupart des théories anthropologiques n'ont pas. Dans les deux chapitres suivants, l'auteur exemplifie et affine ces propositions à partir de cas concrets, tout en introduisant les chevilles autour desquelles le livre s'articule: la nécessité d'étudier les rapports à la place

1. Cf.: La Souche, la cuve et la bouteille. Les rencontres de l'histoire et de la nature dans un aliment: le vin, Paris, Éd. de la MsH, 1988; Les Sociétés et leurs natures, Paris, A. Colin, 1989 ("Anthropologie au présent»); Sociologie comparée du cannibalisme, 1. Proies et captifs en Afrique, 2. La consommation d'autrui en Asie et en Océanie, 3. Ennemis intimes et absorptions équivoques en Amérique, Paris, Presses universitaires de France, 2010-2013; L'Écologie kidnappée, Paris, Presses universitaires de France, 2014. 
des causes, et celle de donner la priorité aux événements et aux perturbations sur les équilibres (structurels et systémiques). Le chapitre IV, enfin, est un plaidoyer en faveur d'un comparatisme révisé, tout en élaborant une approche dialectique de l'observation des rapports au monde. Cela étant dit, il est difficile de rendre justice à cet ouvrage foisonnant, passionné et engagé. Pour le lecteur qui aime être bousculé, le propos est tour à tour fascinant, agaçant, dérangeant ou revigorant. Peut-être que, pour finir, le plus simple est d'appuyer là où l'ouvrage ébranle certains tropismes anthropologiques, parce que c'est également aux anthropologues que Georges Guille-Escuret confie in fine le soin d'utiliser et de discuter son outillage.

C'est un projet matérialiste devenu rare qui supporte l'ouvrage, comme une nécessité de voir aussi les choses du point de vue des sciences, non pas pour construire une hégémonie, mais pour poser une base commune à partir de laquelle contraster et interroger les multiples façons de comprendre et de fabriquer le monde. Cela doit permettre de donner une consistance à la vie sociale, dont on ne se contente plus alors de considérer qu'elle appuie ses représentations et ses actions sur des matières, mais qu'elle se constitue avec elles. L'histoire est centrale. Elle est envisagée dans ses contingences temporelles et spatiales, avec Whitehead contre Braudel et le symbolisme. Les relations ont été évoquées. Elles incitent à penser symétriquement, sans que la subjectivité des uns ne puisse prétendre à définir tout le rapport. Le propos remet également le comparatisme au cœur de l'entreprise

scientifique et interdisciplinaire, mais, en le conceptualisant précisément, il prend ses distances avec la notion floue que, selon Georges Guille-Escuret, on invoque sans la pratiquer. Reste enfin le «cadre de référence», qui doit établir "la marque de la science, son obligation primordiale et la marque de son identité» (p. 59) face à la philosophie, qui n'est contrainte que par la logique. C'est cet objet commun et processuel qui est censé favoriser le dialogue interdisciplinaire là où l'on constate le plus souvent une cohabitation et un empilement d'approches.

En ressuscitant les perdants magnifiques de l'histoire de l'anthropologie et en leur offrant la mise en perspective qui leur faisait défaut, Georges Guille-Escuret pose un jalon déterminant pour la pratique de l'interdisciplinarité. Si on pourra paradoxalement lui reprocher le manque d'exemples interdisciplinaires, quelques choix formels discutables quoique défendables (comme celui d'utiliser un système d'assertions méthodologiques, qui donnent des repères pour la discussion mais résonnent comme une scansion) et une tendance au raccourci que l'isolement de son propos et les mensurations modestes du volume expliquent en partie mais ne justifient pas toujours, l'ouvrage est néanmoins à la hauteur de sa visée. Il est en tout cas essentiel qu'il soit lu et examiné pour ce qu'il est au fond: un grand plaidoyer pour la réouverture de débats fondamentaux.

Léo Mariani 